\title{
Efficient placement of proxies for hierarchical reliable multicast
}

\author{
Liansheng Tan ${ }^{\mathrm{a}, \mathrm{b}, *}, \mathrm{Li}_{\text {Jin }}{ }^{\mathrm{a}}$, Yi Pan ${ }^{\mathrm{c}}$ \\ a Department of Computer Science, Central China Normal University, Wuhan 430079, PR China \\ ${ }^{\mathrm{b}}$ The Research School of Information Sciences and Engineering, The Australian National University, Canberra ACT 0200, Australia

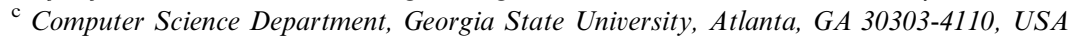

Received 29 September 2006; received in revised form 4 December 2007; accepted 4 December 2007

Available online 14 December 2007

\begin{abstract}
The primary focus of this paper is the design of efficient algorithm for the calculation of solution to the placement of proxies for performance optimization in hierarchical reliable multicast (HRM). Proxy is introduced to localize feedback and retransmission, and therefore significantly depress traffic redundancy and reduce latency. Placement of proxies is a key issue in HRM. This paper theoretically analyzes the performance improvement made by placement of proxies and presents a new approach to optimal partition of a subtree for performance optimization in the placement of a single proxy. Based on this approach, we propose a new algorithm, designed in a greedy fashion. We experimentally compare our new algorithm with several existing heuristic algorithms, and observe that it outperforms in the tradeoff between performance and complexity.
\end{abstract}

(c) 2007 Elsevier B.V. All rights reserved.

Keywords: Hierarchy; Optimal partition; Reliable multicast; Proxy

\section{Introduction}

IP multicast was proposed as an extension to IP unicast service for efficient multipoint communication, composed of an addressing standard, methodologies for users to become members of multicast groups (internet group management protocol), multicast distribution trees (sourcerooted and shared trees), and a multicast routing protocol (protocol independent multicast). IP multicast makes better bandwidth utilization, eliminates traffic redundancy and enables multipoint applications. However, its UDPbased nature makes best-effort delivery and lack of congestion avoidance, resulting in occasional packet loss, duplicate packets, out-of-sequence delivery, even network congestion as multicast group grows.

To improve reliability and scalability of IP multicast, reliable multicast (RM) was proposed with a number of recovery techniques $[8,18,20,28]$, e.g. automatic repeat

\footnotetext{
${ }^{*}$ Corresponding author. Tel.: +61 2 61258679; fax: +61 261258660 .

E-mail addresses: liansheng.tan@rsise.anu.edu.au (L. Tan), pan@cs. gsu.edu (Y. Pan).
}

request (ARQ), forward error correction (FEC) $[23,25]$, and hybrid error control (HEC). This paper concerns with ARQ-based reliable multicast. ARQ uses feedback to detect loss and arrange retransmission, including senderinitiated recovery (using ACK) [1,12,17], and receiver-initiated recovery (using NACK) [5]. However, a number of simultaneous feedbacks would overwhelm the source, incurring implosion problem. Moreover, packet loss on some receivers would expose the others to unnecessary retransmissions, incurring exposure problem [10].

An efficient approach to address these problems is the tree-based hierarchical proxy approach (shown in Fig. 1), dividing a multicast distribution tree (multicast group) into several subtrees (subgroups) to form a hierarchy rooted at the source. A representative node, called proxy, is designed to detect packet loss and arrange retransmission inside each subgroup. Thus the incidence of implosion and exposure are suppressed significantly. Meanwhile, bandwidth is saved, enabling large-scale reliable multicast. Moreover, recovery latency is reduced because retransmission comes from a proxy located much more close than the source. Many existing reliable multicast protocol follow this 


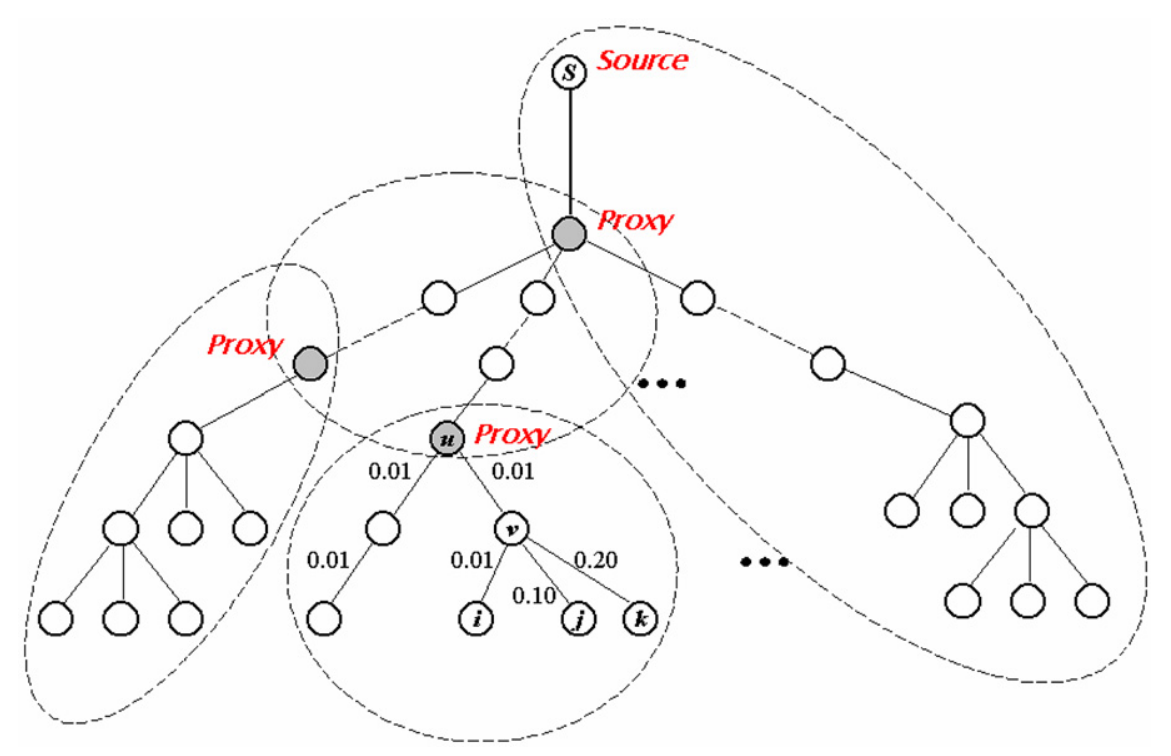

Fig. 1. Tree-based hierarchical proxy approach.

approach, e.g. RMTP [21], LBRM [10], LGMP [19], OTERS [16], TMTP [30], and RMX [6] (a.k.a. "hierarchical reliable multicast" (HRM) [9,15]).

The performance of such approach strongly depends on the underlying multicast tree topology, link loss characteristics and location of proxies. Placement of proxies consumes overhead and bandwidth. Frequent proxy switching may incur excessive packet loss and additional retransmission. Hence, to make the approach achieve optimal performance, appropriate placement of proxies is essential.

The primary focus of this paper is the design of an efficient algorithm for the calculation of an approximate solution to the placement of proxies for optimal performance in HRM. Our goal is to minimize overall traffic required for one-to-many data delivery and reduce recovery latency in HRM. We review the related works in Section 2 and present a system model for HRM with several assumptions in Section 3. In Section 4, we theoretically analyze the performance improvement of HRM obtained from the placement of proxies and introduce several related measures, formulate our goal and converse it into an equivalent issue. We propose our algorithm based on a new approach in Section 5 , then, present a numerical example for illustration in Section 6. We experimentally compare our algorithm with several heuristic algorithms and further analyze these results in Section 7. Finally, we analyze the complexity of our algorithm and conclude the paper in Sections 8 and 9, respectively.

Our contribution is a new approach to optimal partition of a subtree, and a new algorithm for the calculation of solutions to placement of proxies for optimal performance in HRM with a running time of $\mathrm{O}\left(k n\left(1+\delta_{\max }\right)\right)$, where is $\delta_{\max }$ the maximum number of children of any node. Our new algorithm outperforms in the tradeoff between performance and complexity.
The number of proxies, say $\mathrm{k}$, is appointed according to the need or capability of the network. We use the number of total link transmissions for a packet to arrive all receivers correctly to signify the bandwidth required, which we need to minimize to achieve our goal.

\section{Related work and contribution}

We start by reviewing a graph theoretic topic, $k$-median problem [3], to perceive the proxy placement issue.

The $k$-median problem is stated as follows: Given n points, select $k$ of them to be centers, and then assign each input point $j$ to the closest selected center. If $j$ is assigned to a center $i$, a cost $d_{j} c_{i j}$ is incurred. The goal is to select $k$ centers to minimize the sum of the assignment costs. Unfortunately, this problem is proved to be NP-hard in [4]. However, there are constant-factor approximation algorithms for $k$-median problem.

The proxy placement for HRM resembles some variants of the $k$-median problem, and the facility location problem [13], which is NP-hard in graph case, but not in tree case.

In [15], Li et al. proposed a tree-based algorithm, with a running time of $\mathrm{O}\left(n^{3} k^{2}\right)$. It places $k_{\mathrm{i}}$ proxies optimally in each subtree $T_{\mathrm{i}}$, where $\sum_{i} k_{i}=k$ and $\sum_{i} T_{i}=T$, and achieves a global optimal solution finally. However, calculating optimal solution is too computationally intensive to be practical.

More recent studies [11,22], based on evaluation using real traces and topologies, have concluded that a greedy algorithm can provide approximate solutions for optimal performance. The optimal solution provided by tree-based algorithm is usually not as good as the solution found by the greedy algorithm.

Qiu et al. [22] experimentally compared several heuristic algorithms and found a simple greedy algorithm performed the best. The greedy algorithm places the $k$ proxies individ- 
ually within $k$ iterations. Although this algorithm depends upon estimates of client distance and load predictions, it provides approximate solutions at a low computation cost. Moreover, the greedy algorithm is found to be relatively insensitive to errors in these estimates, and viable for use in the general Internet environment with imperfect workload information.

In [13], Krishnan et al. study the optimal placement of web caches. Their goal is to minimize the overall flow or the average delay. In [26], Shah et al. deal with the $k$-median problem in the context of content-based multicast. They define a filter placement problem as a version of $k$ median and provide two algorithms for filter placement to minimize the mean total network bandwidth utilization and mean delivery delay. In [7], Daescu et al. study two NAK-suppressing agent activation problems, and present efficient algorithms for the optimal activation of agents on a multicast tree. In addition, a significant amount of study has been done on the placement of proxies for reliable multicast $[9,14,28,29]$. The main objective of these works is to reduce retransmissions, latency and improve resource utilization. In [9], Sudipto Guha et al. also presents an optimal algorithm in $\mathrm{O}\left(n^{3} k^{2}\right)$ and an approximation algorithm within a chosen $(1+\varepsilon)$ precision from the optimal, in $\mathrm{O}\left(n h k^{2} \log n / \varepsilon\right)$ time, where $h$ is the height of the tree. In [29], Tang et al. studied the QoS-aware placement under replica-aware and replica-blind model, respectively. They experimentally evaluate several heuristic algorithms and propose efficient algorithms for replicas placement under different cost models.

\section{System model and assumptions}

The underlying topology of HRM is tree; therefore, we concentrate on one-to-many applications that reliably transfer information in a one-to-many data dissemination system application in this paper. Our model for HRM features the following characteristics:

- The multicast distribution tree (multicast group) is divided into several subtrees (subgroups) to form a hierarchy rooted at the source.

- The root of each subtree acts as the proxy inside this subgroup, but as an ordinary member inside the upstream subgroup.

- Original packets and parity packets are transmitted in multicast way inside each subgroup.

- Each receiver sends ACK to the proxy inside the subgroup, after receiving a packet successfully.

- Each proxy repeatedly retransmits a packet if not receiving $\mathrm{ACK}(\mathrm{s})$ from one or more receivers inside the subgroup. It sends the next packet after receiving $\mathrm{ACK}(\mathrm{s})$ from all receivers inside the subgroup.

Network bandwidth required for reliable multicast tightly depends on the underlying characteristics of the multicast tree, some necessary information, such as multicast route and link loss probability, must be known. We assume that the topology and loss probability on all links are known. In our model, since the topology of the tree is a relatively static one in time, the realization of this assumption is possible [24,27].

Moreover, we assume that feedback and poll for feedback packet (ACK) are assumed to consume neither bandwidth nor transmission time, and to be transmitted with out loss.

\section{Problem definition and solutions}

In this section, we theoretically analyze the performance improvement obtained from placement of proxies, and propose several related measures, then formulate our goal and converse it into an equivalent issue.

\subsection{Related measures in reliable multicast}

A commonly used measure in the performance analysis of reliable multicast is $E[M]$ that first appeared in [2]. It is defined as the expected number of transmissions per packet from a node required for reliable delivery to all receivers below. $E[M]$ captures the expected amount of workload on the node and affects the network bandwidth used. It strongly depends on the underlying tree topology and loss characteristics.

The paper [2] introduces the probability mass function $P[M \leqslant m]$ and the cumulative distribution function $F_{i}(m)$ to compute $E[M]$ measure in a recursive fashion, starting from the leaves $(l)$, and then the intermediate nodes $(n)$, and finally the source $(s)$.

$$
\begin{aligned}
& F_{i}(m)=P[M(i) \leqslant m], \\
& F_{l}(m)=1-p_{l}^{m}, \\
& F_{n}(m)=\sum_{u=0}^{m-1}\left(\begin{array}{l}
m \\
u
\end{array}\right) p_{n}^{u}\left(1-p_{n}\right)^{m-u} \prod_{k \in c h i l d} F_{k}(m-u), \\
& F_{s}(m)=\prod_{k \in c h i l d} F_{k}(m), \\
& E[M(i)]=\sum_{m=0}^{\infty}\left(1-F_{i}(m)\right),
\end{aligned}
$$

where $M(i)$ is the number of transmissions from node $i$ required for reliable delivery, $p_{n}$ is the loss probability on the link leading to node $n$, and child ${ }_{n}$ is the set of children of node $n$.

Although these formulas are general enough to compute $E[M]$ measure in any case, they are not intuitive and too computationally intensive to operate in practice.

We calculate the $E[M]$ measure by using an approach to find the equivalent link of an arbitrary topology composed of several links in a bottom-up way, which was proposed in [5]. Fig. 2(a) and (b) show the equivalent link of three links in a row or in a star with respect to the $E[M]$ measure.

A multicast tree, the more general topology, can be seen as equivalent to a link as well, with the appropriate loss 
a

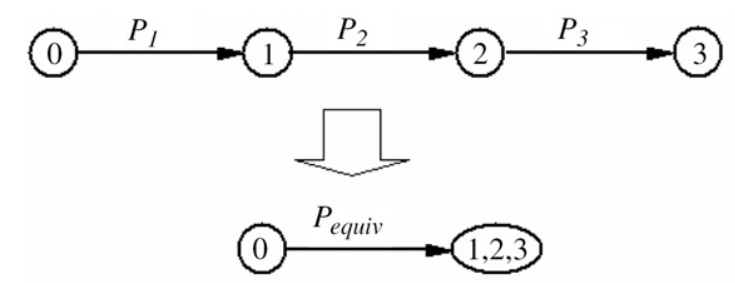

$$
\begin{aligned}
& p_{\text {equiv }}=1-\left(1-p_{1}\right)\left(1-p_{2}\right)\left(1-p_{3}\right) \\
& E[M(0)]=\frac{1}{1-p_{\text {equiv }}}=\frac{1}{\left(1-p_{1}\right)\left(1-p_{2}\right)\left(1-p_{3}\right)}
\end{aligned}
$$
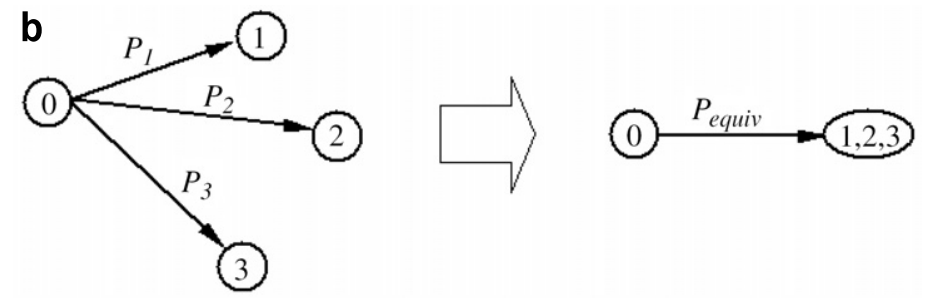

$$
\begin{aligned}
E[M(0)] & =\frac{1}{1-p_{\text {equiv }}}=\sum_{i=1}^{3}\left(\begin{array}{l}
3 \\
i
\end{array}\right)(-1)^{i+1} \frac{1}{1-p^{i}} \\
& =\frac{1}{1-p_{1}}+\frac{1}{1-p_{2}}+\frac{1}{1-p_{3}} \\
& -\frac{1}{1-p_{1} p_{2}}-\frac{1}{1-p_{1} p_{3}}-\frac{1}{1-p_{2} p_{3}} \\
& +\frac{1}{1-p_{1} p_{2} p_{3}}
\end{aligned}
$$
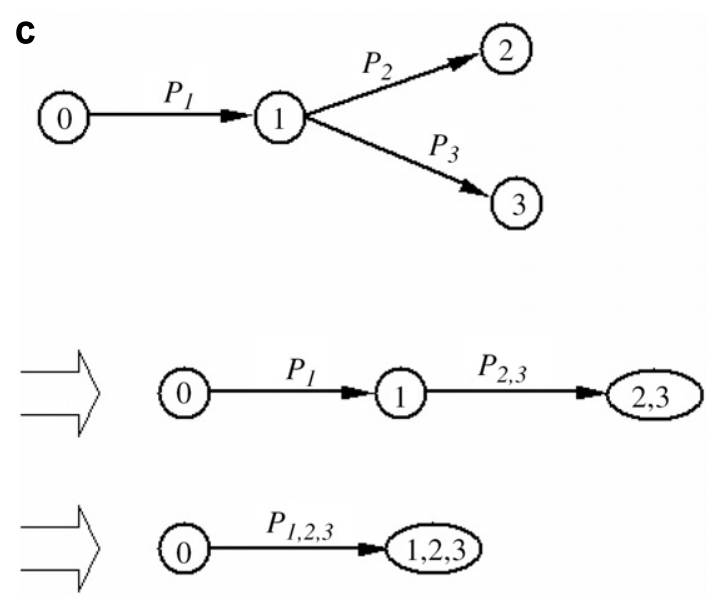

$$
\begin{aligned}
& E[M(1)]=\frac{1}{1-p_{2,3}}=\frac{1}{1-p_{2}}+\frac{1}{1-p_{3}}-\frac{1}{1-p_{2} p_{3}} \\
& E[M(0)]=\frac{1}{1-p_{1,2,3}}=\frac{1}{\left(1-p_{1}\right)\left(1-p_{2,3}\right)}
\end{aligned}
$$

Fig. 2. (a) Equivalent link of three links in a row. (b) Equivalent link of three links in a star. (c) Equivalent link of three links in a tree.

rate $p_{\text {equiv }}$ such that the $E[M]$ seen by the source is the same. Fig. 2(c) shows the equivalent link of three links in a tree with respect to the $E[M]$ measure. Applying those rules from the leaves and proceeding up towards the source, leads to the calculation of $E[M]$ on all nodes in a multicast tree.

However, in reliable multicast, the number of transmissions from a certain node is not always as expected for the affect of the high loss links. Except the proxies, all other intermediate nodes, have to forward all packets received, even the unnecessary retransmissions.
The probability that node $v$ receives a packet correctly from an upstream node $u$ is:

$p(u, v)= \begin{cases}1, & \text { if } v=u ; \\ \prod_{i \in(u, v)}\left(1-p_{i}\right), & \text { if } v \neq u\end{cases}$

where $(u, v)$ is the route between node $u$ and node $v, i$ is an intermediate node on $(u, v)$, with link loss probability $p_{i}$.

For a node $i$, if $i$ is not a proxy, $i$ has to forward all packets received from its proxy, hence the transmissions from $i$ is $E\left[M\left(A P_{i}\right)\right]^{*} p\left(A P_{i}, i\right)$; if $i$ is a proxy, the transmissions from 
$i$ are composed of two parts: one part is the transmissions when $i$ acts as the proxy in its subgroup, that is $E[M(i)]$. The other part is the transmissions when $i$ acts as an ordinary member in the upstream subgroup, that is $E\left[M\left(A P_{i}\right)\right] *$ $p\left(A P_{i}, i\right)$. Therefore, we define the $A[M]$ measure, the actual number of transmissions from an arbitrary node, as follows:

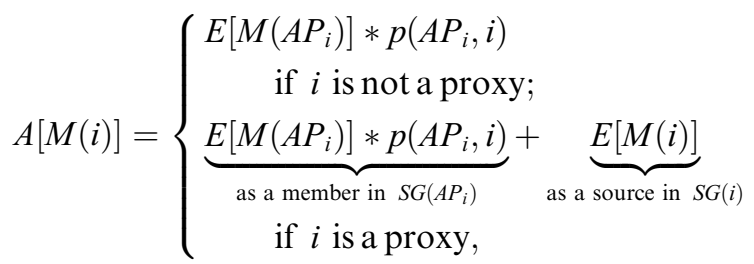

where $A P_{i}$ is the assigned proxy for node $i$, and $S G(i)$ is the subgroup, whose proxy is node $i$.

The contribution of an arbitrary node $i$ to the number of total link transmissions is shown in Table 1 , where $c h_{i}$ is the number of the children of node $i$, and $c h_{i}(j)$ is the number of the children of node $i$, whose assigned proxy is node $j$. Furthermore, there is $c h_{i}=c h_{i}\left(A P_{i}\right)+c h_{i}(i)$.

\subsection{Proxy placement and transmission reduction}

Selecting a node, says node $v$, to be a proxy will incur the change in link transmissions in the subgroup $S G\left(A P_{v}\right)$ (if $A P_{v}$ is the source, $S G\left(A P_{v}\right)$ can be the entire multicast group). Since the whole or a portion of subtree $T_{v}$ is partitioned out from $S G\left(A P_{v}\right)$ to form $S G(V), E\left[M\left(A P_{v}\right)\right]$ will decrease. Meanwhile, since node $v$ changes from an intermediate node to a proxy, unnecessary retransmissions in $S G(V)$ will decrease as well. Hence, the reduction in the number of total link transmissions caused by this placement can be divided into two parts: (i) the reduction in the number of link transmissions inside $S G\left(A P_{v}\right)$ but outside $S G(V)$ due to the decrease of $E\left[M\left(A P_{v}\right)\right]$; (ii) the reduction in the number of link transmissions inside $S G(V)$ due to the change of the role of node $v$.

We define the reduction in total link transmissions caused by placing node $v$ as the proxy of the entire subtree $T_{v}$ as follows:
Table 1

Contribution of an arbitrary node $I$ to total link transmissions

\begin{tabular}{ll}
\hline $\begin{array}{l}\text { Type of } \\
\text { node } i\end{array}$ & Contribution to the total link transmissions \\
$\begin{array}{l}\text { Intermediate } \\
\text { node }\end{array}$ & $A[M(i)] * c h_{i}=E\left[M\left(A P_{i}\right)\right] * p\left(A P_{i}, i\right) * c h_{i}$ \\
Proxy & $A[M(i)] * c h_{i}=E\left[M\left(A P_{i}\right)\right] * p\left(A P_{i}, i\right) * c h_{i}\left(A P_{i}\right)$ \\
& $+E[M(i)] * c h_{i}(i)$ \\
\hline
\end{tabular}

all links in subtree $T_{u}$ are given. We can obtain $E[M(u)]=$ 1.384122. Suppose node $v$ is going to be placed as a proxy. There are three solutions to partition $T_{u}$ into two subgroups.

Solution 1: If we partition the entire subtree $T_{v}$ out from $T_{u}$, there are

$$
\begin{aligned}
E^{\prime}[M(u)] & =1.040212, E^{\prime}[M(v)]=1.347999, \operatorname{Reduction}(v) \\
& =1.435605 .
\end{aligned}
$$

Almost all the algorithms for proxy placement partition multicast tree in the same way as that used in solution 1, namely, assigning nodes to its closest proxy. However, it is not always the best choice, sometimes even the worst choice.

Solution 2: If we partition $T_{u}$ into two subgroups as shown in Fig. 3(a), we can obtain

$$
\begin{aligned}
E^{\prime}[M(u)] & =1.158491, E^{\prime}[M(v)]=1.25, \operatorname{Reduction}(v) \\
& =1.465041 .
\end{aligned}
$$

Solution 3: If we partition $T_{u}$ into two subgroups as shown in Fig. 3(b), we can obtain

$$
\begin{aligned}
E^{\prime}[M(u)] & =1.050123, E^{\prime}[M(v)]=1.340703, \operatorname{Reduction}(v) \\
& =1.719290 .
\end{aligned}
$$

We can see that if we place node $v$ as a proxy, partition subtree $T_{u}$ into two subgroups as shown in Fig. 3(b) can obtain the maximum reduction in total link transmissions.

$$
\begin{aligned}
\operatorname{Reduction}(v)= & \underbrace{\sum_{i \in S G(v)}\left(E\left[M\left(A P_{v}\right)\right] * p\left(A P_{v}, v\right)-E^{\prime}[M(v)]\right) * p(v, i) * c h_{i}(v)}_{\text {reduction inside subgroup } S G(v)} \\
& +\underbrace{\sum_{j \in\left\{S G\left(A P_{v}\right)-S G(v)\right\} \cup\{v\}}\left(E\left[M\left(A P_{v}\right)\right]-E^{\prime}\left[M\left(A P_{v}\right)\right]\right) * p\left(A P_{v}, j\right) * c h_{j}\left(A P_{v}\right)}_{\text {reduction outside subgroup } S G(v)}
\end{aligned}
$$

where $E[M(i)]$ and $E^{\prime}[M(i)]$ are the values of the $E[M]$ measure seen on node $i$ before and after the placement, respectively.

To demonstrate the reduction incurred by the placement of proxies, consider the multicast tree in Fig. 1, with the assumption that node $u$ is a proxy and loss probabilities on
The essence of maximizing the reduction is making best effort to separate the high loss links from other links. It is a principle of appropriate partition of multicast tree. It is also the basic idea of our algorithm. Therefore, in our algorithm, we do not always assign a certain node to its closest proxy, but according to the need of obtain- 

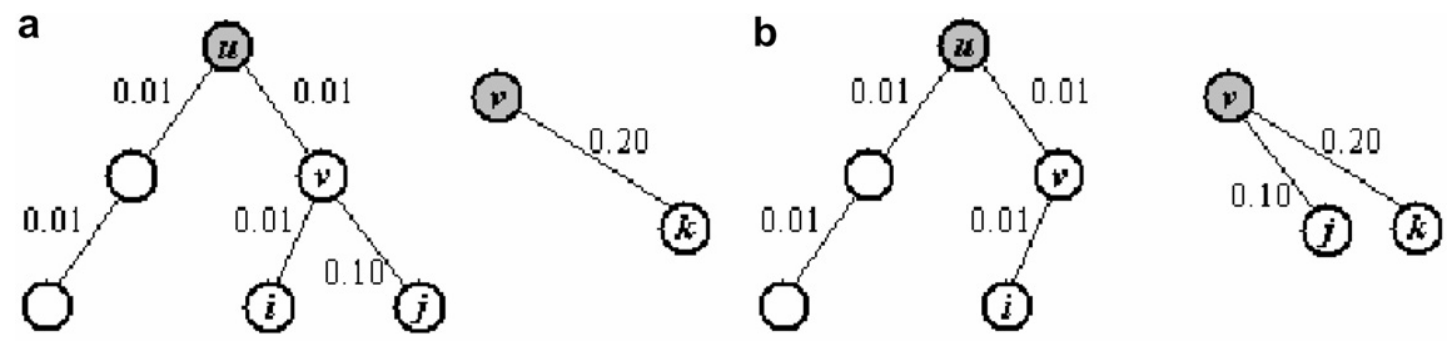

Fig. 3. Two choices for partition of subtree $T_{v}$.

ing the maximum reduction. It is an important difference between our algorithm and other algorithms for proxy placement.

\subsection{Objective formulation and conversion}

With a set $P$ of proxies, the number of total link transmissions per packet required for reliable delivery in a multicast tree $T_{s}$ is:

total link transmissions $=\sum_{i \in P} \sum_{v \in S G(i)} E[M(i)] * p(i, v) * c h_{v}(i)$.

Therefore, we formulate our objective as:

$\min \sum_{i \in P} \sum_{v \in S G(i)} E[M(i)] * p(i, v) * c h_{v}(i)$.

Notice that, the number of original total link transmissions per packet for reliable delivery without the support of the proxies is $\sum_{i \in T_{s}} E[M(s)] * p(s, i) * c h_{i}$, which is a fixed value. Accordingly, we convert our objective into an equivalent and formulate it as:

$\max \left(\sum_{i \in T_{s}} E[M(s)] * p(s, i) * c h_{i}-\sum_{i \in P} \sum_{v \in S G(i)} E[M(i)] * p(i, v) * c h_{v}(i)\right)$,

which means to maximize the reduction in the number of total link transmissions caused by the placement of proxies.

\section{Greedy-MRS algorithm}

Our algorithm tries to minimize the number of total link transmissions by maximizing the reduction caused by the placement of proxies. An optimal group partition algorithm is proposed to optimal partition the subtree rooted at a potential node to incur the maximum reduction. Based on this algorithm, we design Greedy-MRS for proxy placement in a greedy fashion. The node that can achieve the maximum reduction is placed as proxy in single iteration to achieve local optimal solution, thus, a global suboptimal solution is achieved finally.

\subsection{Optimal group partition achieves maximum reduction}

We have shown in Section 4.2 that assigning nodes to its closest proxy is not always the best choice, sometimes even the worst choice. To attack this problem, we propose an optimal group partition algorithm shown in Fig. 4 to find out the optimal solution to partition a subtree rooted at a potential node that can be placed as a proxy to incur the maximum reduction.

In this algorithm, we thought a subtree $T_{u}$ as the union of several branches. Each branch $T_{u, v}$ corresponds with a child $v$ of node $u$. The metric $\boldsymbol{d}_{\boldsymbol{i}}=\boldsymbol{E}[\boldsymbol{M}(\boldsymbol{i})] /\left(1-\boldsymbol{p}_{i}\right)$ reflects the degree of congestion in branch $T_{u, i}$. We arrange all children of node $u$ according to this metric in descending order. Correspondingly, all branch of subtree $T_{u}$ are arranged according to their degree of congestion in descending order as well.

Then, we initial $\operatorname{Reduction}(u)=0$, and investigate all children of node $u$ orderly. We investigate the No. $k$ child by computing Reduction' $(u)$ incurred by partition the union of the first $k$ branches out as a subgroup. If Reduc$\operatorname{tion}^{\prime}(u)>\operatorname{Reduction}(u), \operatorname{Reduction}(u)=\operatorname{Reduction}^{\prime}(u)$ and the investigation continues. Once Reduction' $(u)<$ Reduc$\operatorname{tion}(u)$ in the No. $k$ investigation, the investigation stops. Reduction $(u)$ is node $u$ 's maximum reduction incurred if being a proxy. If Reduction $(u)$ is the greatest among the maximum reductions of all nodes, node $u$ is placed to be a proxy, and the optimal partition of subtree $T_{u}$ is partition the union of the first $k-1$ branches out as a new subgroup. And we use $\boldsymbol{B}_{\boldsymbol{u}}$ to denote the set of the selected children of node $u$, corresponding to the branches that should be partitioned out.

Consider the multicast tree in Fig. 1 for example once again. When we calculate Reduction $(v)$, we first arrange all $i \in$ child $_{v}$ according to $d_{i}$ in descending order. Since that $d_{i}=1.010101, d_{j}=1.111111$, and $d_{k}=1.25$, we obtain the ordered children set $c h i l d_{v}^{\prime}=\{k, j, i\}$. Then, we initial $\operatorname{Reduction}(v)=0$, and investigate all children of node $v$ orderly.

We first investigate the first node in child ${ }_{v}^{\prime}$, node $k$. As calculated in Section 4.2, Reduction' $(v)$ incurred by partition the branch $T_{v, k}$ out is 1.465041 . Since that Reduc$\operatorname{tion}^{\prime}(v)>\operatorname{Reduction}(v), \operatorname{Reduction}(v)=1.465041$ and the investigation continues.

Then, we investigate the first two nodes in child ${ }_{v}^{\prime}$, node $k$ and $j$. Reduction' $(v)$ incurred by partition the union $T_{v, k} \cup T_{v, j}$ out is 1.719290. Since that Reduc$\operatorname{tion}^{\prime}(v)>\operatorname{Reduction}(v)$, Reduction $(v)=1.719290$ and the investigation continues. 


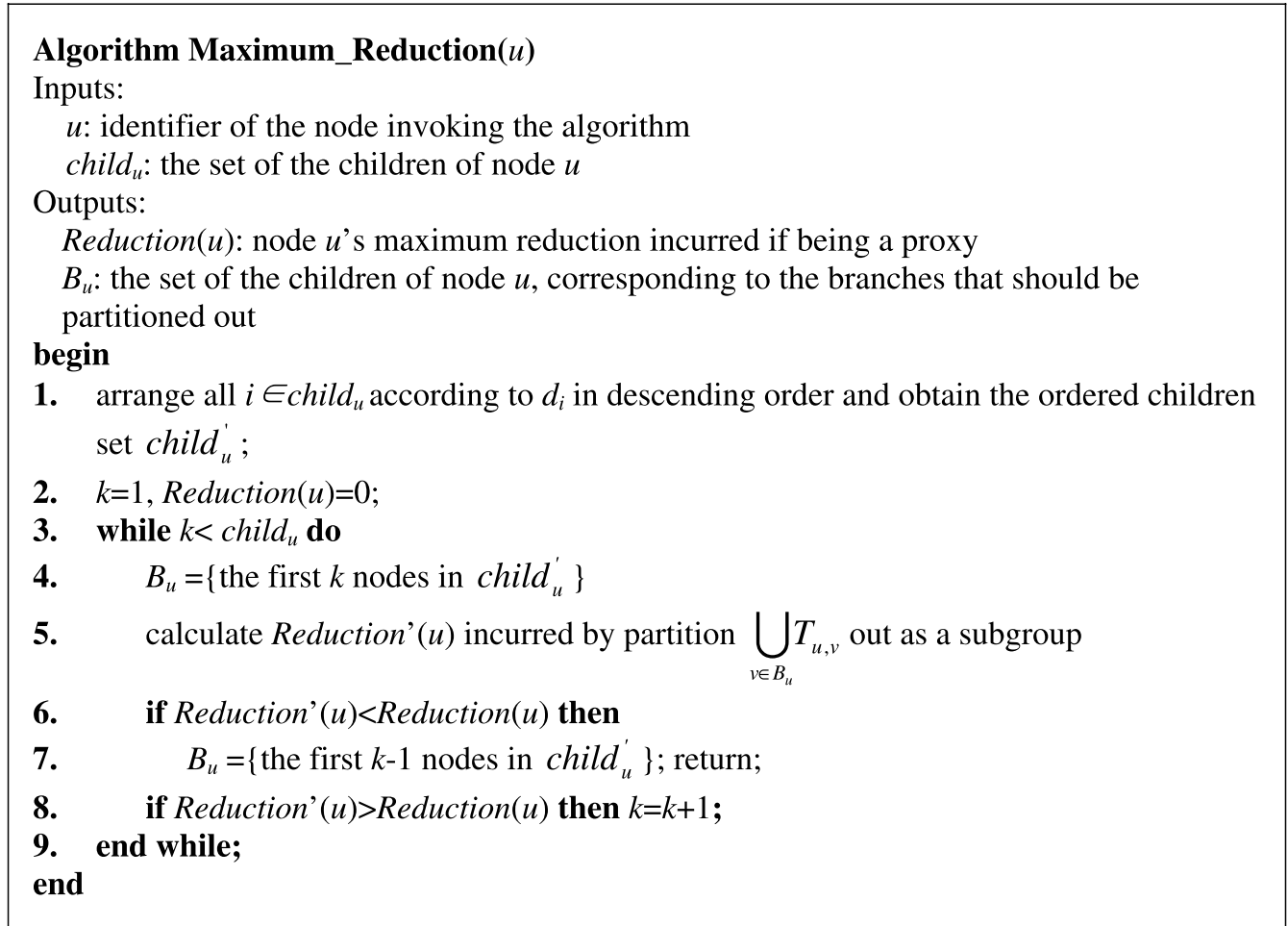

Fig. 4. Optimal group partition algorithm.

Finally, we investigate all three children. Reduction' $(v)$ incurred by partition the entire subtree $T_{v}$ out is 1.435605. Since that Reduction' $(v)<$ Reduction (v), Reduc$\operatorname{tion}(v)=1.719290$ at last and the optimal partition of subtree $T_{v}$ is partition the union $T_{v, k} \cup T_{v, j}$ out as a new subgroup. In other words, $B_{v}=\{j, k\}$.

\subsection{Algorithm outline}

Based on the optimal group partition algorithm, we propose our algorithm for proxy placement, called Greedy$M R S$, as shown in Fig. 5.

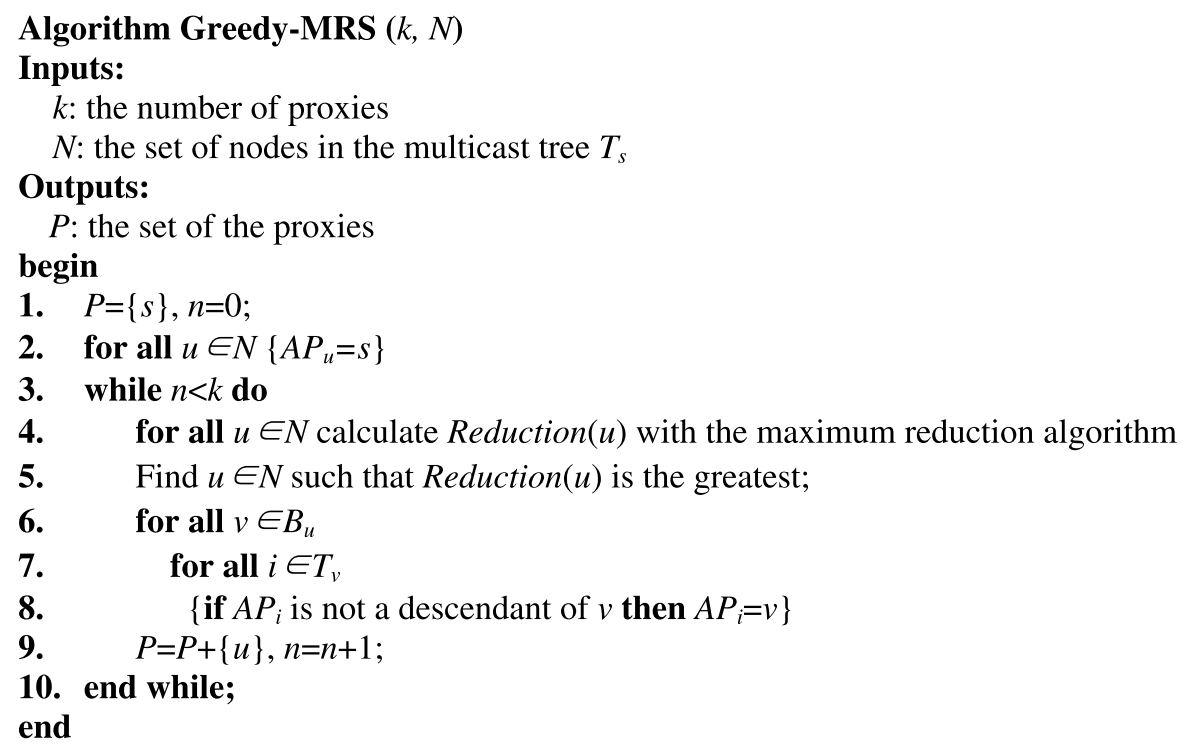

Fig. 5. Greedy-MRS algorithm. 
Our algorithm for proxy placement is designed in a greedy fashion that the $k$ proxies are placed one by one. The only appropriate node that can achieve the maximum reduction is placed to be a proxy in single iteration, achieving a local optimal solution. The same procedure is repeated until the $k$ proxies are placed. Thus, a global suboptimal solution is achieved finally. Different from other heuristics, Greedy$M R S$ does not assign nodes to its closest proxy, but according to the need of obtaining the maximum reduction.

\section{A numerical example illustration}

We consider a network topology given in Fig. 6(a) and take the tree for example to demonstrate the placement of proxies using our algorithm. We purpose placing $k=3$ proxies (the root is a default proxy) in this network, which is composed of $n=15$ nodes (including the root being the multicast source).

The initial status of this network is shown in Table 2.

Tables 3-5 show the reduction of each node computed during the placement of each time, respectively.
The reduction computation for selecting the first proxy proceeds in the entire multicast group. From Table 3, we can see that Reduction(1) is the greatest among that of all nodes, hence node 1 is placed as the first proxy. To gain the maximum reduction, node $2,4,5,6,9,10,11,12,13$, and 14 switch their assigned proxy from node 0 to node 1 .

After the first proxy is placed, the multicast tree is divided into two subgroups: $\{0,1,3,7,8\}$ and $\{1,2,4,5,6,9,10,11$, $12,13,14\}$. The reduction computation for selecting the second proxy proceeds in these two subgroups. From Table 4, we can see that Reduction(5) is the greatest among that of all nodes, hence node 5 is placed as the second proxy. To gain the maximum reduction, node 13 and 14 switch their assigned proxy from node 1 to node 5 .

After the second proxy is placed, the multicast tree is divided into three subgroups: $\{0,1,3,7,8\},\{1,2,4,5$, $6,9,10,11,12\}$ and $\{5,13,14\}$. The reduction computation for selecting the third proxy proceeds in these three subgroups. From Table 5, we can see that Reduction(2) is the greatest among that of all nodes, hence node 2 is placed as the third proxy. To gain the maximum reduc-
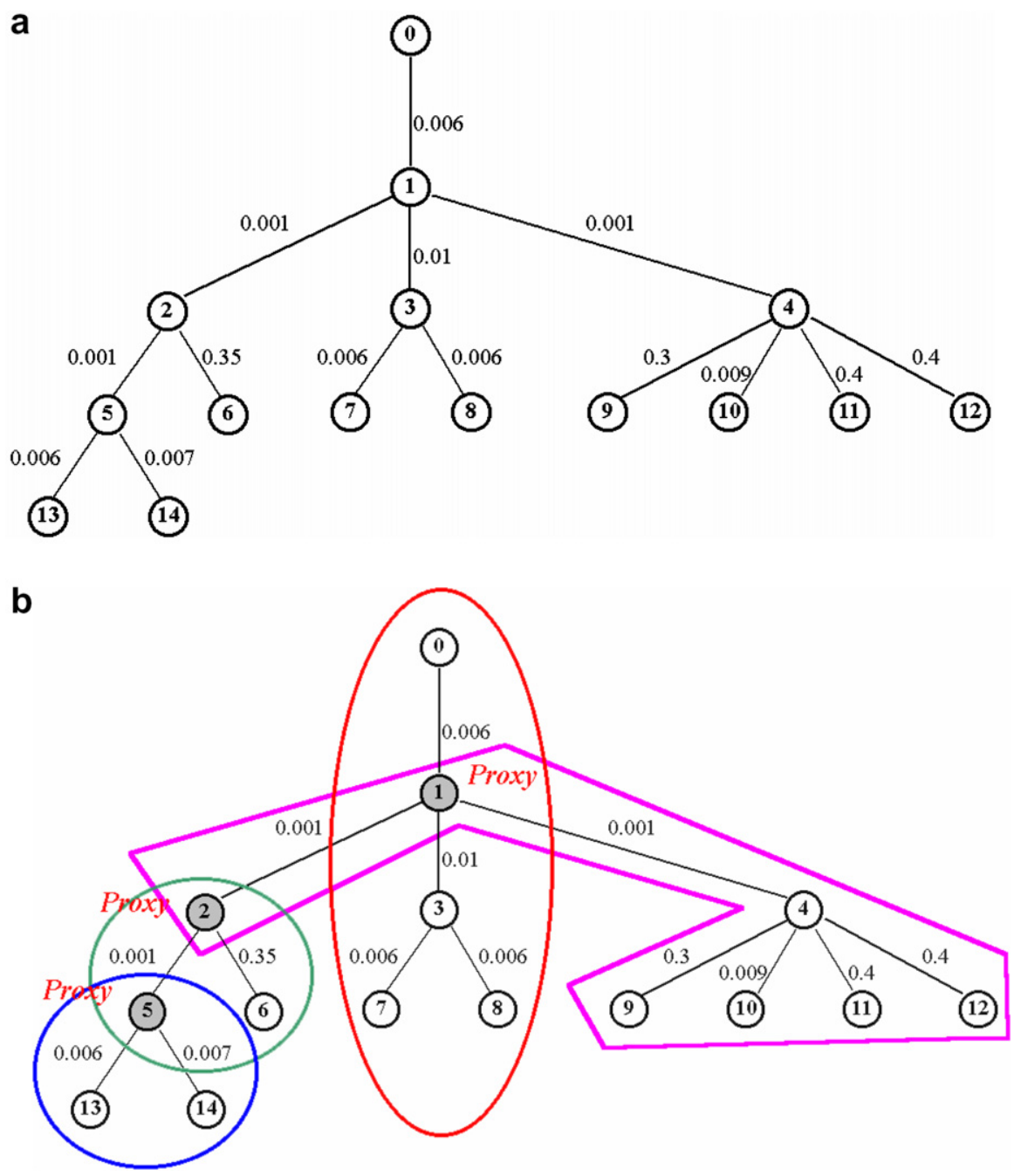

Fig. 6. (a) An example topology to demonstrate the placement of proxies using Greedy-MRS algorithm. (b) The solution to the placement of three proxies in the example topology using Greedy-MRS algorithm. 
tion, nodes 5 and 6 switch their assigned proxy from node 1 to node 2 .

Finally, the three selected proxies are node 1, 2, and 5, and the multicast tree is divided into four subgroups: $\{0$, $1,3,7,8\},\{1,2,4,9,10,11,12\},\{2,5,6\}$ and $\{13,14\}$, as shown in Fig. 6(b).

Table 2

Initial status of the network

\begin{tabular}{ll}
\hline Node $i$ & $A P_{i}$ \\
\hline 1 & 0 \\
2 & 0 \\
3 & 0 \\
4 & 0 \\
5 & 0 \\
6 & 0 \\
7 & 0 \\
8 & 0 \\
9 & 0 \\
10 & 0 \\
11 & 0 \\
12 & 0 \\
13 & 0 \\
14 & 0 \\
\hline
\end{tabular}

Table 3

Placement of the first proxy

\begin{tabular}{rlll}
\hline Node $i$ & $A P_{i}$ & $B_{i}$ & Reduction $(i)$ \\
\hline 1 & 0 & $\{4,2\}$ & 2.599442 \\
2 & 1 & $\{5,6\}$ & 0.512564 \\
3 & 0 & $\{7,8\}$ & 1.291231 \\
4 & 1 & $\{13,14\}$ & 0.000000 \\
5 & 1 & & 1.315901 \\
6 & 1 & & 0.000000 \\
7 & 0 & & 0.000000 \\
8 & 0 & & 0.000000 \\
9 & 1 & & 0.000000 \\
10 & 1 & & 0.000000 \\
11 & 1 & & 0.000000 \\
12 & 1 & & 0.000000 \\
13 & 1 & & 0.000000 \\
14 & 1 & & 0.000000 \\
\hline
\end{tabular}

Table 4

Placement of the second proxy

\begin{tabular}{llll}
\hline Node $i$ & $A P_{i}$ & $B_{i}$ & Reduction $(i)$ \\
\hline 1 & 0 & $\{3\}$ & 0.016296 \\
2 & 1 & $\{5,6\}$ & 0.512564 \\
3 & 0 & $\{7,8\}$ & 0.012231 \\
4 & 1 & $\{13,14\}$ & 0.000000 \\
5 & 1 & & 1.315902 \\
6 & 1 & & 0.000000 \\
7 & 0 & & 0.000000 \\
8 & 0 & & 0.000000 \\
9 & 1 & & 0.000000 \\
10 & 1 & & 0.000000 \\
11 & 1 & & 0.000000 \\
12 & 1 & & 0.000000 \\
13 & 5 & & 0.000000 \\
14 & 5 & & 0.000000 \\
\hline
\end{tabular}

Table 5

Placement of the third proxy

\begin{tabular}{clll}
\hline Node $i$ & $A P_{i}$ & $B_{i}$ & Reduction $(i)$ \\
\hline 1 & 0 & $\{3\}$ & 0.016296 \\
2 & 1 & $\{5,6\}$ & 0.256410 \\
3 & 0 & $\{7,8\}$ & 0.012231 \\
4 & 1 & & 0.000000 \\
5 & 2 & & 0.000000 \\
6 & 2 & & 0.000000 \\
7 & 0 & & 0.000000 \\
8 & 0 & & 0.000000 \\
9 & 1 & & 0.000000 \\
10 & 1 & & 0.000000 \\
11 & 1 & & 0.000000 \\
12 & 1 & 0.000000 \\
13 & 5 & & 0.000000 \\
14 & 5 & & 0.000000 \\
\hline
\end{tabular}

\section{Simulation and analysis}

In this section, we perform simulations to evaluate the performance of Greedy-MRS against three other algorithms:

(1) Optimal: an algorithm based on the tree-based algorithm proposed by $\mathrm{Li}$ et al. [15], and shown to find an optimal placement in [9] in a running time of $\mathrm{O}\left(n^{3} k^{2}\right)$

(2) Hot Spot: an algorithm attempting to place proxies near the nodes generating the greatest load. It sorts the $n$ potential nodes according to the amount of traffic generated within their vicinity and places the top $k$ nodes as the $k$ proxies.

(3) Random: an algorithm randomly choosing the $k$ proxies among the $n$ potential nodes.

\subsection{Random simulation scenarios}

In our simulations, the topology of multicast tree is randomly generated, and loss probabilities on all links are given randomly as well. Each simulation run is parameterized by

(i) Multicast group size: the number of members in the multicast group.

(ii) Loss characteristics of multicast tree (H:M:L): the ratio among the amounts of links categorized according to their loss probability. This parameter captures the degree of congestion in the network. For instance, $\mathrm{H}: \mathrm{M}: \mathrm{L}=1: 3: 6$ means that of all links, $10 \%$ are high loss links, 30\% are mid loss links, and 60\% are low loss links. In our simulations, links in more than $20 \%$ loss are deemed to be high loss, links in low than $5 \%$ loss are deemed to be low loss, and other links are deemed to be mid loss. The definition of the categorization is not that strict, however, it will not affect the justice of the evaluation, since that all algorithms deal 
with the same multicast tree, the same loss characteristics in each simulation run.

(iii) Proxies percentage (\#Proxies): the percentage of the multicast group to be placed as proxies. For instance, if the multicast group size is 1000, \#Proxies $=10 \%$ means that 100 nodes are proxies.

\subsection{Performance measures}

The major purpose for deploying proxies is to reduce the retransmission, hence we consider a transmission reduction ratio $(T R R)$ to be the main performance measure. We also use the average latency to measure the effectiveness of the placement of proxies.

- Transmission reduction ratio. We define this measure as follows:

TRR

$=\frac{\sum_{i \in T_{s}} E[M(s)] * p(s, i) * c h_{i}-\sum_{i \in P} \sum_{v \in S G(i)} E[M(i)] * p(i, v) * c h_{v}(i)}{\sum_{i \in T_{s}} E[M(s)] * p(s, i) * c h_{i}}$

- Average latency. We define the latency of a receiver node as its distance (hop counts) to its proxy. The Average latency is average of the latencies of all receiver nodes.
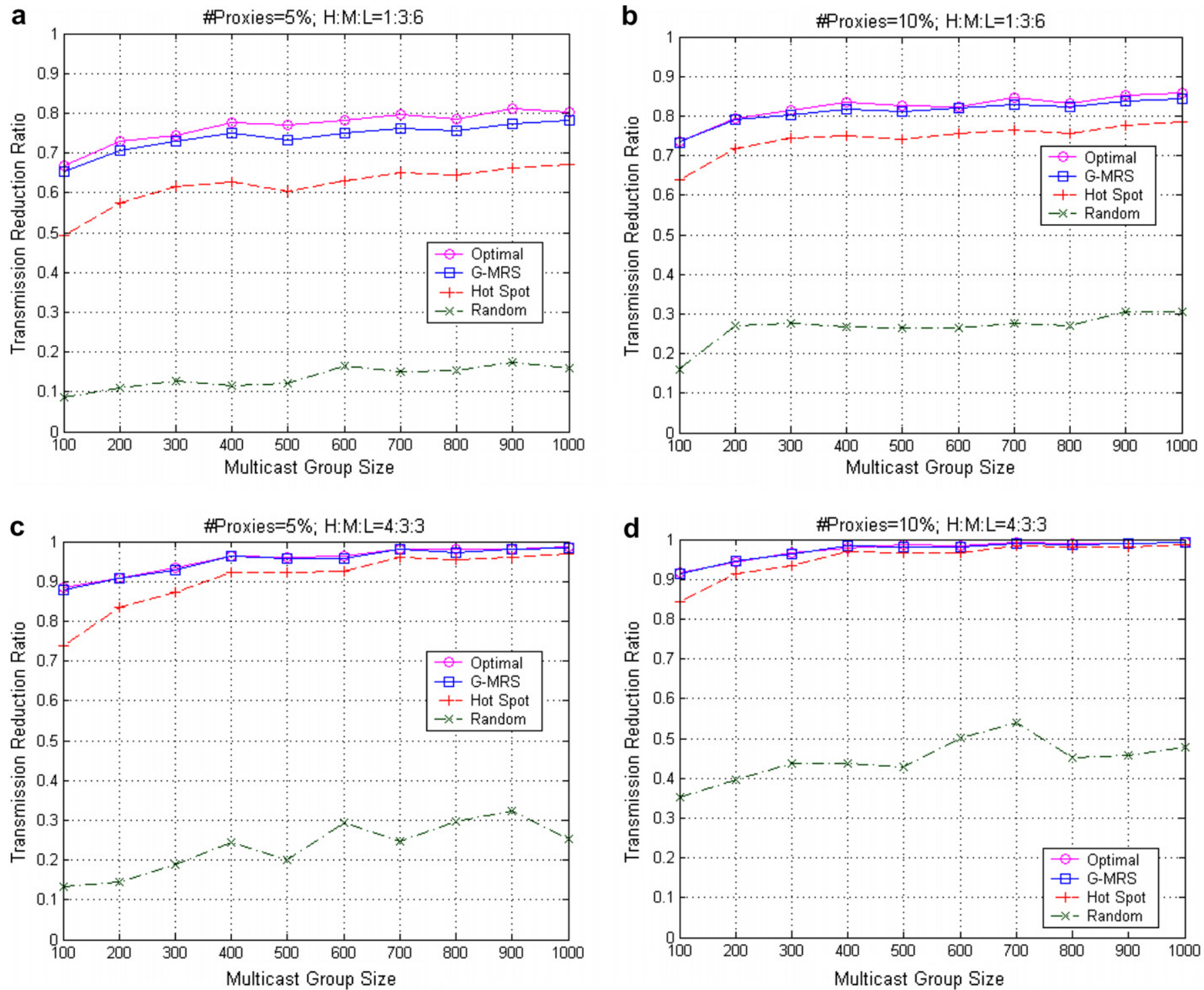

Fig. 7. (a) TRR ( $5 \%$ nodes in multicast group are proxies, and high-loss links:mid-loss links:low-loss links $=1: 3: 6)$. (b) TRR (10\% nodes in multicast group are proxies, and high-loss links:mid-loss links:low-loss links =1:3:6). (c) TRR (5\% nodes in multicast group are proxies, and high-loss links:mid-loss links:low-loss links $=4: 3: 3)$. (d) TRR (10\% nodes in multicast group are proxies, and high-loss links:mid-loss links:low-loss links $=4: 3: 3)$. (e) Average latency ( $5 \%$ nodes in multicast group are proxies, and high-loss links:mid-loss links:low-loss links =1:3:6). (f) Average latency (10\% nodes in multicast group are proxies, and high-loss links:mid-loss links:low-loss links $=1: 3: 6) .(\mathrm{g})$ Average latency $(5 \%$ nodes in multicast group are proxies, and high-loss links:mid-loss links:low-loss links = 4:3:3). (h) Average latency (10\% nodes in multicast group are proxies, and high-loss links:mid-loss links:low-loss links $=4: 3: 3)$. 

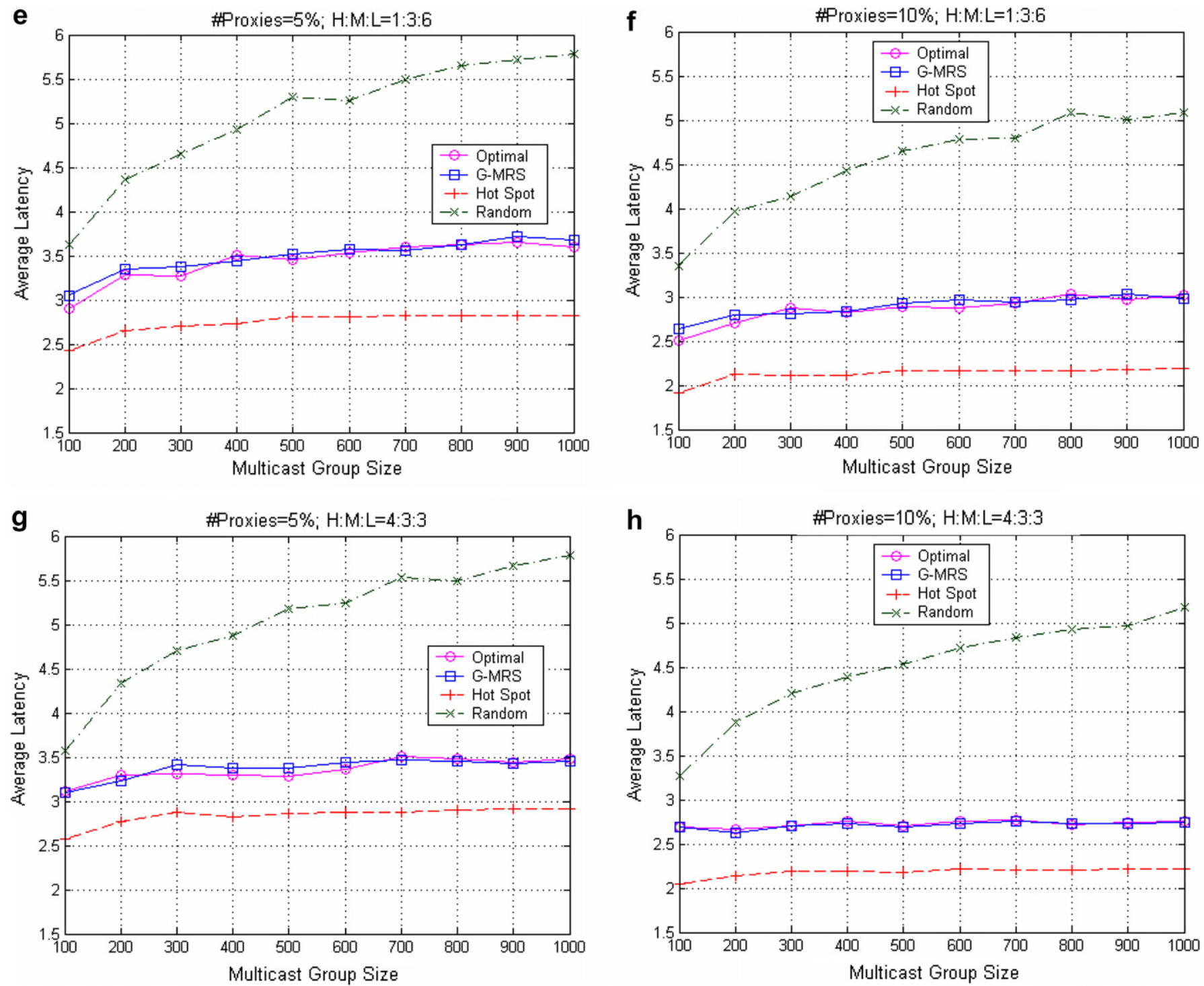

Fig. 7 (continued)

\subsection{Simulation results}

We vary the multicast group size from 100 nodes to 1000 nodes, and place $5 \%$ and $10 \%$ nodes as proxies, respectively, in both $\mathrm{H}: \mathrm{M}: \mathrm{L}=1: 3: 6$ and $\mathrm{H}: \mathrm{M}: \mathrm{L}=4: 3: 3$ case, respectively, as well.

For each data point in our simulation chart, 50 tree topologies are generated randomly parameterized by the multicast group size and the loss characteristics of multicast tree. For each multicast tree, we run the four algorithms to evaluate their performance with the two measures, respectively. The performance of a certain algorithm for a certain measure under the given parameters is the average performances of this algorithm in the simulations with the 50 tree topologies randomly generated with these parameters.

Simulation results are shown from Fig. 7(a-d). From these results, we found that:
(1) Transmission reduction ratio: The transmission reduction ratio increases as the number of proxies increases with all four algorithms. And this measure can be affected by the multicast group size as well that it increases as the multicast group size increases, although this phenomena is not that strict. When the degree of congestion is light, we can see from Figs. 7)(a) and (b) that Greedy-MRS exhibits a slight lower transmission reduction ratio when compared to the Optimal algorithm. Still, the performance of Greedy$M R S$ for this measure is higher than that of the Hot Spot algorithm, and much higher than that of the Random algorithm. When the degree of congestion is high, we can see from Figs. 7(c) and (d) that the performance of Greedy-MRS for the transmission reduction ratio measure is very close to that of the Optimal algorithm, and still higher and much higher than that of the Hot Spot algorithm and that of the Random algorithm, respectively. 
(2) Average latency: From Fig. 7(e-h), we can see that increasing the multicast group size does not have an obvious effect on the average latency to most of the algorithms, except the Random algorithm.

The performance of Greedy-MRS for the average latency measure is very close to that of the Optimal algorithm as well. Its latency is much smaller than that of the Random algorithm, especially as multicast group sizes increase, but greater than that of the Hot Spot algorithm. It is because that we do not always assign a certain node to the closest proxy (See Fig. 8).

\section{Complexity analysis}

The complexity of maximum reduction calculation for each node is $\mathrm{O}\left(\delta_{\max }\right)$, where $\delta_{\max }$ is the maximum number of children of any node. The complexity of placing the node with the maximum reduction to be a proxy is $\mathrm{O}(n)$, where $n$ is the number of nodes in the multicast group. Therefore, the complexity of placing a single proxy with our Greedy-MRS algorithm is $\mathrm{O}\left(n\left(1+\delta_{\max }\right)\right)$. Finally, the complexity of our Greedy-MRS algorithm for the placement of $k$ proxies is $\mathrm{O}\left(k n\left(1+\delta_{\max }\right)\right)$.

We tabulated the computational time of various algorithms for proxy placement in Table 6 .

\section{Implementation vista}

Greedy-MRS algorithm can be introduced into network routers (or layer-3 switches) that support HRM protocols. Once proxies are needed, these routers would cooperate with each other to select the appropriate ones to be proxies. The routers, acting as proxies, can efficiently separate the subgroups that encounter high packet loss from other subgroups (shown in Fig. 9), and therefore eliminate redundant traffic and suppress the incidence of congestion.

However, acting as proxies and supporting Greedy-MRS require more storage and processing power, and therefore more expensive to operate than normal routers. The more routers support Greedy-MRS in HRM networks, the better solution Greedy-MRS provides. The solution can perform best if all routers support Greedy-MRS (shown in Fig. 9). The technique that how these routers communicate with each other after being implemented by this new algorithm could be a topic for future study.

Table 6

Comparison of computational time of various algorithms

\begin{tabular}{llll}
\hline Optimal & Greedy-MRS & Hot spot & Random \\
\hline $\mathrm{O}\left(n^{3} k^{2}\right)$ & $\mathrm{O}\left(k n\left(1+\delta_{\max }\right)\right)$ & $\mathrm{O}\left(n^{2}+k n\right)$ & $\mathrm{O}(n k)$ \\
\hline
\end{tabular}

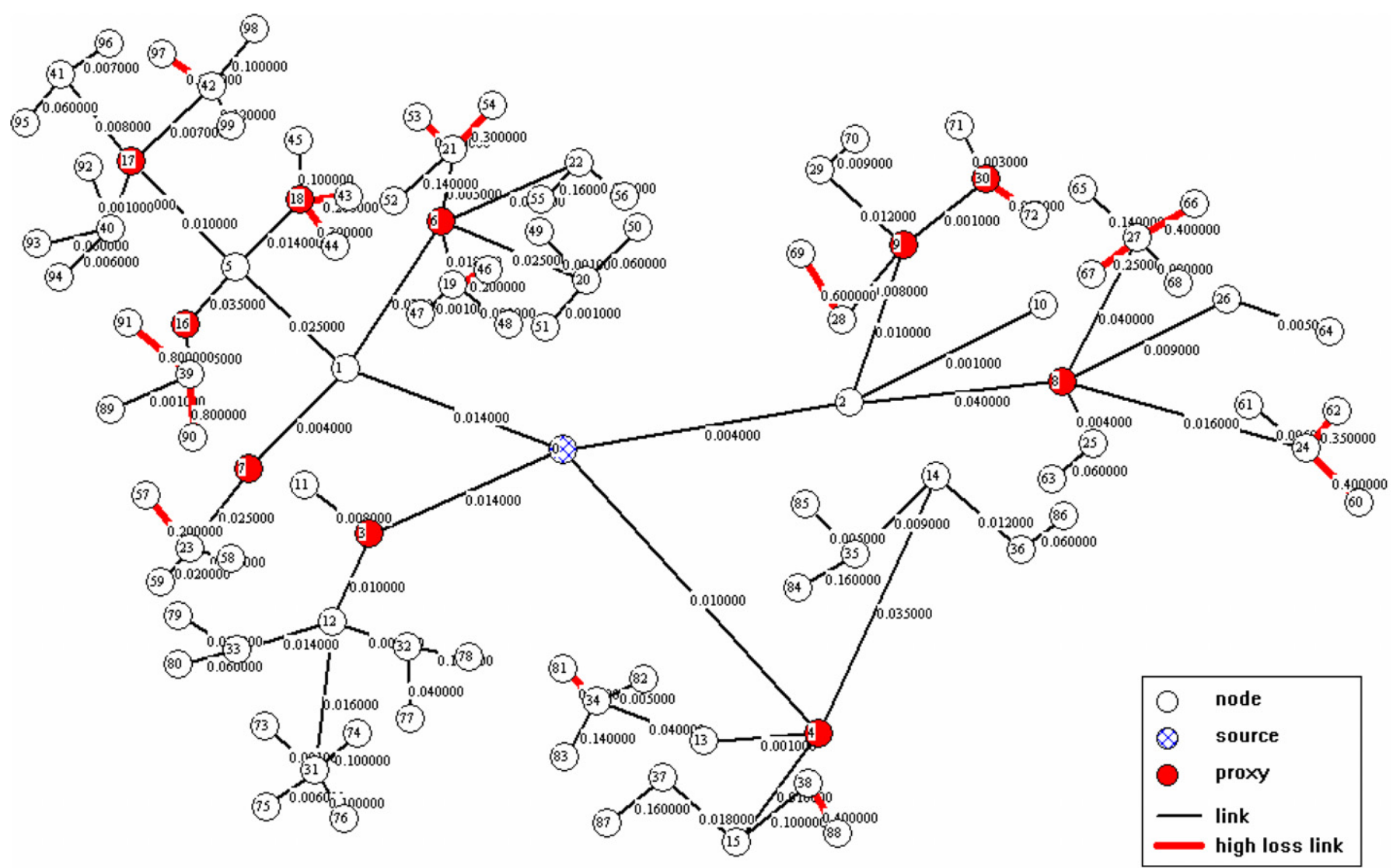

Fig. 8. The solution of placement of $10 \%$ proxies in a 100 nodes scenario using Greedy-MRS algorithm. 

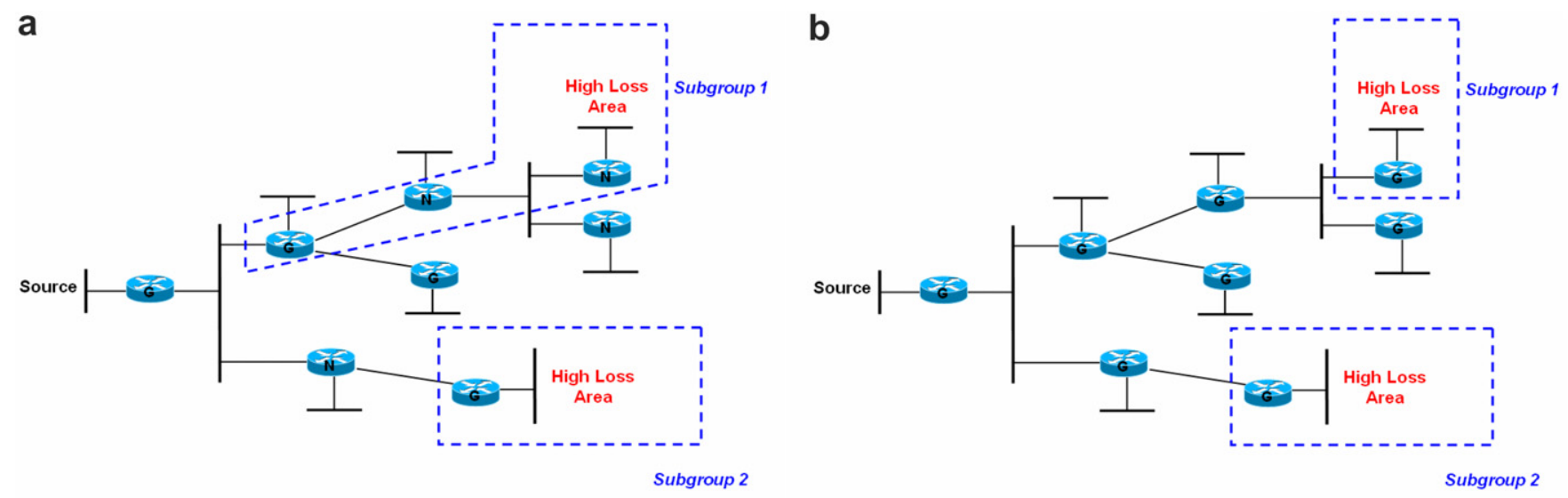

Normal Router

Normal Router 6 Greedy-MRS Router

Fig. 9. Different solutions provided by Greedy-MRS according to router capability: (a) not all routers support Greedy-MRS; (b) all routers support Greedy-MRS.

\section{Conclusions}

In this paper, we theoretically analyze the performance improvement earned from placement of proxies and observe that the practice of assigning clients to the closest proxy, followed by almost all existing algorithms, is not always the best choice, sometimes even the worst. Therefore we present a new approach to optimal partition of a subtree, maximizing the improvement in the placement of a single proxy. Further, we propose our new greedy fashion algorithm. We experimentally compare it with several existing heuristic algorithms, and observe that our algorithm outperforms in the tradeoff between performance and complexity. We believe it is much more well-founded and efficient than other heuristic algorithms, but lower in complexity.

\section{Acknowledgement}

This work was partially supported by the Program NCET-05-0673 from the Chinese Ministry of Education and partially by the Australian Research Council.

\section{References}

[1] F. Baccelli, A. Chaintreau, Z. Liu, Scalability of reliable group communication using overlays, in: Proc. IEEE INFOCOM '04, Hong Kong, China, March 2004.

[2] P. Bhagwat, P. Mishra, S. Tripathi, Effect of topology on performance of reliable multicast communication, in: Proc. IEEE INFOCOM'94, Toronto, Ontario, Canada, 1994, pp. 602-609.

[3] M. Charikar, S. Guha, E. Tardos, D. Shmoys, A constant-factor approximation algorithm for the k-median problem, in: Proc. 31st Annual Symposium on the Theory of Computing, Atlanta, Georgia, USA, 1999, pp. 1-10.

[4] P. Crescenzi and V. Kann, A compendium of NP optimization problems, August, 1998, Available at: http://www.nada.kth.se/viggo/ problemlist/compendium.html.
[5] A.M. Costello, S. McCanne, Search party: using randomcast for reliable multicast with local recovery, in: Proc. IEEE INFOCOM'99, vol. 3, New York, NY, USA,1999, pp. 1256-1264.

[6] Y. Chawathe, S. McCanne, E.A. Brewer, RMX: reliable multicast for heterogeneous networks, in: Proc. IEEE INFOCOM'00, Tel Aviv, Israel, vol. 2, March 2000, pp. 795-804.

[7] O. Daescu, R. Jothi, B. Raghavachari, K. Sarac, Optimal placement of NAK-suppressing agents for reliable multicast: A partial deployment case, in: Proc. 19th ACM Symposium on Applied Computing (SAC), Nicosia, March 2004, pp. 334-338.

[8] S. Floyd, V. Jacobson, C.G. Liu, S. McCanne, L. Zhang, A reliable multicast framework for light-weight sessions and application level framing, IEEE/ACM Transactions on Networking 5 (6) (1997) 784 803 .

[9] S. Guha, A. Markopoulou, F. Tobagi, Hierarchical reliable multicast: performance analysis and optimal placement of proxies, Computer Communications 26 (April) (2003) 2070-2081.

[10] H. Holbrook, S. Singhal, D. Cheriton, Log-based receiver-reliable multicast for distributed interactive simulation, in: Proc. ACM SIGCOMM'95, Cambridge, MA, USA, 1995, pp. 328-341.

[11] S. Jamin, C. Jin, A. Kurc, D. Raz, Y. Shavitt, Constrained mirror placement on the internet, in: Proc. IEEE INFOCOM'01, Anchorage, Alaska, USA, vol. 1, April 2001, pp. 31-40.

[12] S.K. Kasera, J. Kurose, D. Towsley, A comparison of server-based and receiver-based local recovery approaches for scalable reliable multicast, in: Proc. IEEE INFOCOM'98, San Francisco, CA, USA, vol. 3,1998, pp. 988-995.

[13] P. Krishnan, D. Raz, Y. Shavitt, The Cache Location problem, IEEE Transactions on Networking 8 (5) (2000) 568-582.

[14] L. Lao, J.H. Cui and M. Gerla, A scalable overlay multicast architecture for large-scale applications, Technical Report, Department of Computer Science, University of California, July 2004. Available at: http://www.cs.ucla.edu/NRL/hpi/AggMC/papers.

[15] B. Li, M.J. Golin, G.F. Italiano, X. Deng, K. Sohraby, On the optimal placement of web proxies in the internet, in: Proc. IEEE INFOCOM'99, New York, NY, USA, vol. 3, 1999, pp. 1282-1290.

[16] D. Li, D. Cheriton, OTERS: a reliable multicast protocol, in: Proc. of ICNP'98, Austin, TX, USA, 1998, pp. 237-245.

[17] K.W. Lee, S. Ha, V. Bharghavan, IRMA: a reliable multicast architecture for the internet, in: Proc. IEEE INFOCOM'99, New York, NY, USA, vol. 3,1999, pp. 1274-1281.

[18] M.S. Lacher, J. Nonnenmacher, E.W. Biersack, Performance comparison of centralized versus distributed error recovery for reliable 
multicast, IEEE/ACM Transactions on Networking 8 (2) (2000) 224 239. April.

[19] M. Hofmann, Impact of virtual group structure on multicast performance, in: Proc. of the 4th International COST 237 Workshop, Lisboa, Portugal, 1997, pp. 165-180.

[20] G. Papadopoulos, G.M. Parulkar, G. Varghese, An error control scheme for large-scale multicast applications, in: Proc. IEEE INFOCOM'98, San Francisco, CA, USA, vol. 3, 1998, pp. 1188-1196.

[21] S. Paul, K.K. Sabnani, Reliable multicast transport protocol (RMTP), IEEE Journal of Selected Areas in Communications (1997).

[22] L. Qiu, V.N. Padmanabhan, G.M. Voelker, On the placement of web server replicas, in: Proc. IEEE INFOCOM'01, Anchorage, Alaska, USA, vol. 3, April 2001, pp. 1587-1596.

[23] D. Rubenstaein, S. Kasera, D. Towsley, J. Kurose, Improving reliable multicast using active parity encoding services (APES), in: Proc. IEEE INFOCOM'99, New York, NY, USA, vol. 3, 1999, pp. 1248-1255.

[24] S. Ratnasamy, S. McCanne, Inference of multicast routing trees and bottleneck bandwidths using end-to-end measurements, in: Proc. IEEE INFOCOM'99, New York, NY, USA, vol. 1, 1999, pp. 353-363.

[25] D. Rubenstaein, D. Towsley, J. Kurose, Real-time reliable multicast using proactive forward error correction, Technical Report, Department of Computer Science, University of Massachusetts, March 1998.

[26] R. Shah, R. Jain, F. Anjun, Efficient dissemination of personalized information using content-based multicast, in: Proc. IEEE INFOCOM'02, New York, NY, 2002.

[27] D. Towsley, Network tomography using end-to-end multicast measurements, in: Proc. ACM NGC’00, 2000, pp. 93-101.

[28] X. Tang, J. Xu, On replica placement for QoS-aware content distribution, in: Proc. IEEE INFOCOM'04, Hong Kong, China, March 2004.

[29] R. Yavatkar, J. Griffioen, M. Sudan, Reliable dissemination protocol for interactive collaborative applications, in: Proc. ACM MULTIMEDIA'95, Boston, MA, USA, 1995, pp. 333-344.

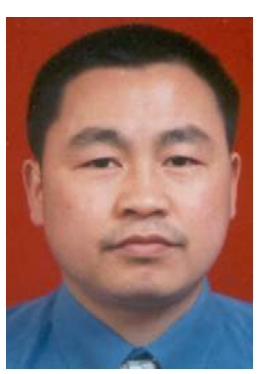

Liansheng Tan is with Research School of Information Sciences and Engineering, The Australian National University, Canberra ACT 0200, Australia. He received his $\mathrm{PhD}$ degree from Loughborough University in UK in 1999 . He has done research in computer communication network in School of Information Technology and Engineering at University of Ottawa, Ontario, Canada as a postdoctoral research fellow and a visiting research scientist in 2001. During 2002 till 2006, he worked at Department of Computer Science, Central China Normal University, China as a professor and Head of Department. During that period, he also held a number of visiting research positions at Loughborough University, University of Tsukuba, City University of Hong Kong and University of Melbourne. He has served as an Associate Editor of Dynamics of Continuous, Discrete \& Impulsive Systems (Series B: Applications \& Algorithms), and as an editor of International Journal of Communication Systems. He has published over seventy referred papers in international journals and conferences. His research interests include modeling, congestion control analysis and performance evaluation of computer communication networks, resource allocation and management of wireless and wireline networks and routing and transmission control protocols.

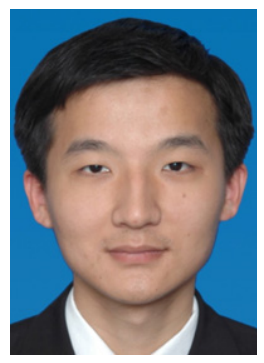

Li Jin was born in Wuhan, China in 1981. He received the B.S. and M.E. degrees in Computer Science from Central China Normal University, China in 2003 and 2006, respectively. Since August 2006, he has been with Shanghai Baosight Software Co., Ltd. as an information security engineer. He is working on network security and information security. His research interests include wireless networks, network security, and distributed systems.

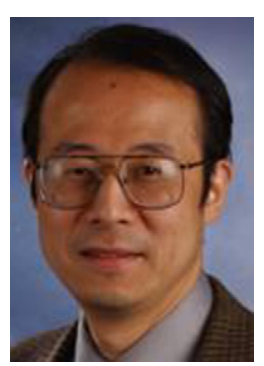

Yi Pan received his B.Eng. and M.Eng. degrees in computer engineering from Tsinghua University, China, in 1982 and 1984, respectively, and his $\mathrm{PhD}$ degree in computer science from the University of Pittsburgh, USA, in 1991. Currently, he is the chair and a full professor in the Department of Computer Science at Georgia State University, USA. His research interests include high performance computing, networking and bioinformatics. He has published more than 100 journal papers with over 30 papers published in various IEEE journals. In addition, he has published over 150 papers in refereed conferences. He has also authored/edited over 33 books (including proceedings). He has served as an editor-in-chief or editorial board member for 15 journals including 5 IEEE Transactions. Dr. Pan has delivered over 10 keynote speeches at international conferences. 